\title{
Interaction-Induced Huge Magnetoresistance in a High Mobility Two-Dimensional Electron Gas
}

\author{
L. Bockhorn*, I. V. Gornyi ${ }^{\dagger}$, D. Schuh**, W. Wegscheider ${ }^{*}$ and R. J. Haug* \\ *Institut für Festkörperphysik, Leibniz Universität Hannover, D-30167 Hannover, Germany \\ $\dagger$ Institut für Nanotechnologie, Karlsruher Institut of Technology, D-76021 Karlsruhe, Germany \\ ** Institut für Experimentelle und Angewandte Physik, Universität Regensburg, D-93053 Regensburg, Germany \\ $\stackrel{\dagger}{+}$ ETH Zürich, Switzerland
}

\begin{abstract}
A strong negative magnetoresistance is observed in a high-mobility two-dimensional electron gas in a $\mathrm{GaAs} / \mathrm{Al}_{0.3} \mathrm{Ga}_{0.7} \mathrm{As}$ quantum well. We discuss that the negative magnetoresistance consists of a small peak induced by a combination of two types of disorder and a huge magnetoresistance explained by the interaction correction to the conductivity for mixed disorder.
\end{abstract}

Keywords: Fractional Quantum Hall Effect, Negative Magnetoresistance, Electron Interaction Correction PACS: 73.43.Qt, 73.23.Ad, 72.10.-d

\section{INTRODUCTION}

The increased mobility of the two-dimensional electron gas (2DEG) has allowed not only the observation of the fractional quantum Hall effect (FQHE)[1],[2] at low magnetic fields but also many new effects. While the mobility and quality of the samples increases the electron electron interaction becomes more important for the magnetotransport at low magnetic fields.

We observe a strong negative magnetoresistance around zero magnetic field which can be divided into two regions. In the present proceeding we study this two regions of the strong negative magnetoresistance, a peak around zero magnetic field and a huge magnetoresistance at larger fields.

\section{SAMPLE INFORMATION}

Our samples were cleaved from a wafer of high-mobility $\mathrm{GaAs} / \mathrm{Al}_{0.3} \mathrm{Ga}_{0.7}$ As quantum well grown by molecularbeam epitaxy. The quantum well is $\mathrm{Si}$-doped from both sides and has a width of $30 \mathrm{~nm}$. The $2 \mathrm{DEG}$ is located $150 \mathrm{~nm}$ beneath the surface and has an electron density of $n_{e}=3.2 \cdot 10^{11} \mathrm{~cm}^{-2}$ and a mobility of $\mu_{e}=$ $11.9 \cdot 10^{6} \mathrm{~cm}^{2} / \mathrm{Vs}$. The specimens are Hall bars with a total length of $1.2 \mathrm{~mm}$, a width of $w=200 \mu \mathrm{m}$ and a potential probe spacing of $l=275 \mu \mathrm{m}$. The Hall geometries were defined by photolithography and wet etching. We structured an additional layer of $600 \mathrm{~nm}$ PMMA between the Hall bar and the metallic top-gate to avoid leakage currents. Our measurements were performed in a dilution refrigerator with a base temperature of $20 \mathrm{mK}$. The magnetotransport measurements were carried out by us- ing low-frequency $(13 \mathrm{~Hz})$ lock-in technique.

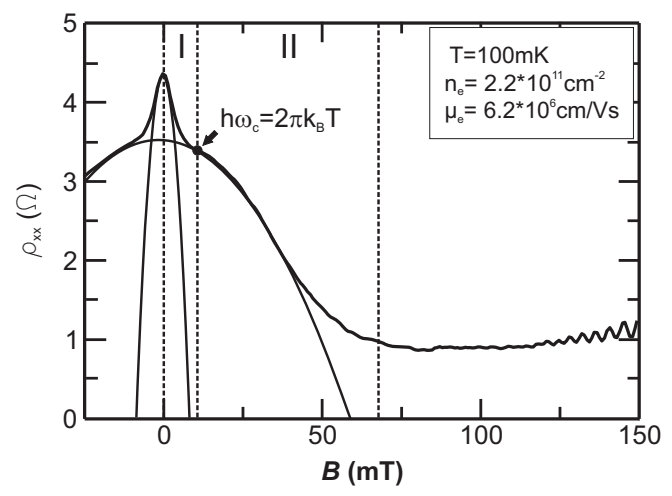

FIGURE 1. The longitudinal resistivity $\rho_{x x}$ vs. the magnetic field $B$. The strong negative magnetoresistance is divided into two sections.

\section{PEAK AROUND ZERO MAGNETIC FIELD}

The strong negative magnetoresistance consist of two regions (Fig. 1). The first region (I) contains a peak around zero magnetic field. The huge magnetoresistance decreases with $B^{2}$ and is fitted by a parabola. In previous experiments we observed that the curvature of the peak is left unchanged by increasing the temperature to $1 \mathrm{~K}$. This temperature independence of the peak is a sign for the absence of weak localization. Also for different electron densities the curavture of the peak is left unchanged.

In accordance with Mirlin et al. [6] we assume that the peak is induced by an interplay of smooth disorder 
and rare strong scatteres. We consider remote scatterers with a smooth random potential and the transport relaxation time $\tau_{s m}$. In the magnetic field range of the peak the cyclotron radius is $R_{c}<10 \mu \mathrm{m}$. The transport scattering time $\tau_{S}$ of randomly distributed strong scatteres also includes scattering with the specimens' edges. The density of the strong scatterers $n_{s}$ can be determined by the curvature of the peak.

\section{HUGE MAGNETORESISTANCE}

The second region (II) of the strong negative magnetoresistance (Fig. 1) contains the huge magnetoresistance (HMR). Previous publictions reported on a strong negative magnetoresistance, which depends strongly on the temperature [3, 4]. The HMR vanished by increasing the temperature. Above $1 \mathrm{~K}$ we observed only the peak around zero magnetic field. The HMR becomes more pronounced by decreasing the electron density, hence the HMR depends also strongly on the electron density.

We examine the electron interaction correction to the conductivity in the situation of a long-range fluctuation potential and in the regime of ballistic transport [5]-[9] to discribe the HMR. In accordance with Gornyi et al. [9] we assume a model of mixed disorder for the characterization of the HMR. The electron interaction induced correction to the conductivity considering the model of mixed disorder is expressed by

$$
\rho_{x x}=\rho_{0}-\rho_{0} \frac{c_{0}}{2 n_{e}^{2} \pi^{2}} \sqrt{\frac{1}{\hbar T \tau k_{B}}} \cdot \alpha \cdot B^{2}
$$

with $c_{0}=0.276$. The additional factor $\alpha$ is in accordance with Gornyi et al. [9] expressed by $\alpha=4\left(\tau_{s m} / \tau\right)^{-1 / 2}$ with $\tau_{s m}=\left(k_{F} d\right)^{2} \tau_{q}$ depending on the quantum scattering time $\tau_{q}$.

From the electron interaction induced correction the curvature is expected to depend on $T^{-1 / 2}$. The HMR is fitted by a parabola for different temperatures, FIG. 2 shows the resulting curvature of the parabola vs. $T^{-1}$. We observed the expected temperature dependence for temperatures below $400 \mathrm{mK}$. Above $400 \mathrm{mK}$ the curvature is approximately proportional to $T^{-1}$ as long as the HMR is observable.

A discrepancy between our meausrement and theory is observed. The factor $\alpha$ determined from the curvature of the HMR is larger than the expected value.

A possible orgin of the discrepancy between theory and experiment is that the quantum scattering time $\tau_{q}$ is dominated by smooth disorder. We know from the peak around zero magnetic field that there should be also a influence of strong scatterers.

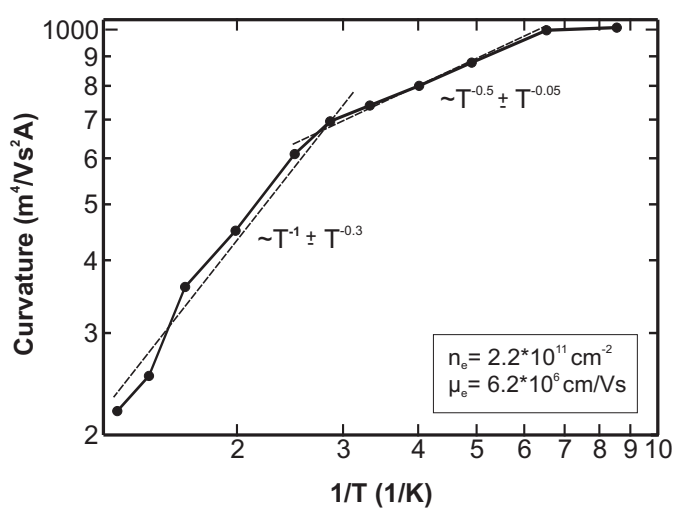

FIGURE 2. The curvature of the HMR vs. $\mathrm{T}^{-1}$.

\section{CONCLUSION}

The strong negative magnetoresistance consists of a peak and the huge magnetoresistance. The huge magnetoresistance depends strongly on the temperature and electron density. We introduce an additional factor $\alpha$ to fit the huge magnetoresistance considering the electron interaction correction. The peak around zero magnetic field is expressed by the interplay of two typs of disorder.

This work was supported by the Cluster of Excellence QUEST.

\section{REFERENCES}

1. D. C. Tsui, H. L. Stormer, and A. C. Gossard, Phys. Rev. Lett. 48, 1559 (1982).

2. R. B. Laughlin, Phys. Rev. Lett. 50, 1395 (1983).

3. L. Bockhorn, P. Barthold, D. Schuh, W. Wegscheider, and R. J. Haug, Phys. Rev. B 83, 113301 (2011).

4. A. T. Hatke, M. A. Zudov, J. L. Reno, L. N. Pfeiffer, and K. W. West, Phys. Rev. B 85, 081304 (2012)

5. M. A. Paalanen, D. C. Tsui, and J. C. M. Hwang, Phys. Rev. Lett. 51, 2226 (1983).

6. A. D. Mirlin, D. G. Polyakov, F. Evers, and P. Wölfle, Phys. Rev. Lett. 87, 126805 (2001).

7. I. V. Gornyi and A. D. Mirlin, Phys. Rev. Lett. 90, 076801 (2003).

8. L. Li, Y. Y. Proskuryakov, A. K.Savchenko, E. H. Linfield, and D. A. Ritchie, Phys. Rev. Lett. 90, 076802 (2003).

9. I. V. Gornyi and A. D. Mirlin, Phys. Rev. B 69, 045313 (2004). 
AIP Conference Proceedings is copyrighted by AIP Publishing LLC (AIP). Reuse of AIP content is subject to the terms at: http://scitation.aip.org/termsconditions. For more information, see http://publishing.aip.org/authors/rights-and-permissions. 